Correction

\title{
Correction: Cohl, H.S.; Costas-Santos, R.S.; Ge, L. Terminating Basic Hypergeometric Representations and Transformations for the Askey-Wilson Polynomials Symmetry 2020, 12, 1290
}

\author{
Howard S. Cohl ${ }^{1, *}$, Roberto S. Costas-Santos ${ }^{2}$ iD and Linus Ge ${ }^{3}$ \\ 1 Applied and Computational Mathematics Division, National Institute of Standards and Technology, \\ Mission Viejo, CA 92694, USA \\ 2 Departamento de Física y Matemáticas, Universidad de Alcalá, 28871 Alcalá de Henares, Spain; \\ rscosa@gmail.com \\ 3 Department of Mathematics, University of Rochester, Rochester, NY 14627, USA; lge6@u.rochester.edu \\ * Correspondence: howard.cohl@nist.gov
}

Received: 18 November 2020; Accepted: 24 November 2020; Published: 21 December 2020

The authors wish to make the following corrections to their paper [1]:

1. In the line immediately following (15), replace $q a b c=\operatorname{def}$ with $q^{1-n} a b c=d e f$.

2. Replace the two Equations (35) and (36) with the following four equations, which should then be listed as (35)-(38), respectively (note that this will push all the equation numbers originally starting from (37) to whatever their equation numbers used to be plus an additional two):

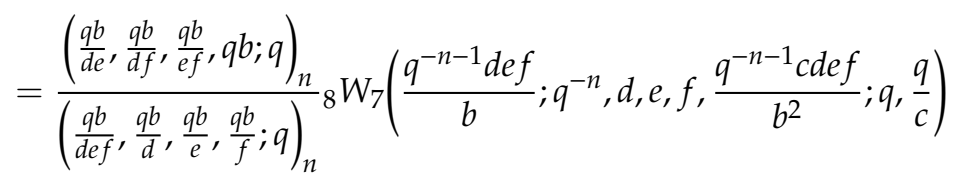

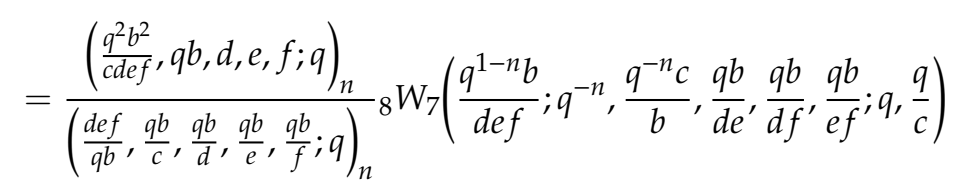

$$
\begin{aligned}
& =\frac{\left(\frac{q^{2} b^{2}}{c d e f}, q b ; q\right)_{n}}{\left(\frac{q b}{c}, \frac{q^{2} b^{2}}{d e f} ; q\right)_{n}} W_{7}\left(\frac{q b^{2}}{d e f} ; q^{-n}, \frac{q b}{d e}, \frac{q b}{d f}, \frac{q b}{e f}, c ; q, \frac{q^{n+1} b}{c}\right) \\
& =q^{\left(\begin{array}{c}
n \\
2
\end{array}\right)}\left(-\frac{q b}{c}\right)^{n} \frac{\left(\frac{q b^{2}}{d e f}, \frac{q b}{e f}, \frac{q b}{d e}, \frac{q b}{d f}, q b, c ; q\right)_{n}}{\left(\frac{q b^{2}}{d e f} ; q\right)_{2 n}\left(\frac{q b}{c}, \frac{q b}{d}, \frac{q b}{e}, \frac{q b}{f} ; q\right)_{n}} \\
& \times_{8} W_{7}\left(\frac{q^{-2 n-1} d e f}{b^{2}} ; q^{-n}, \frac{q^{-n} d}{b}, \frac{q^{-n} e}{b}, \frac{q^{-n} f}{b}, \frac{q^{-n-1} c d e f}{b^{2}} ; q, \frac{q^{n+1} b}{c}\right)
\end{aligned}
$$

3. In the proof of Corollary 3, replace ". Then," with ", setting $\theta \mapsto-\theta$ where necessary. Then,".

4. Replace (44) and (45), respectively (with the updated numbers these will become (46) and (47)) with:

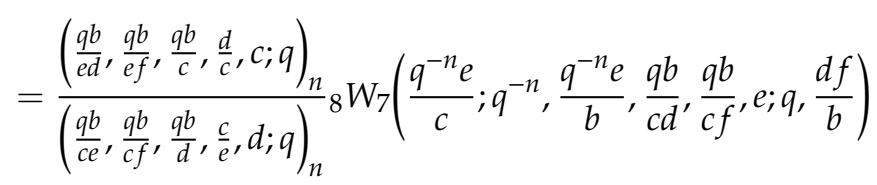




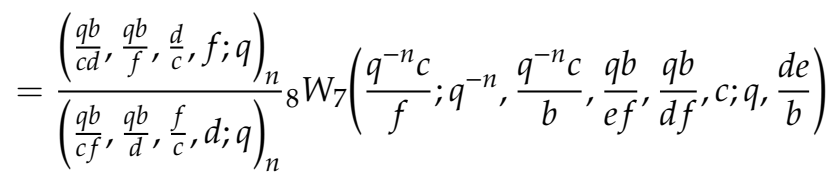

5. Replace the text in Remark 5 (note that we refer to the updated equation numbers) as: "Other sets of parameter interchange transformations can be obtained by considering all permutations of the symmetric parameters $c, d, e, f$ in (35), (36), (38), respectively. However, one can see that these are equivalent to the above Corollary 5 by replacing:

$$
\begin{gathered}
(b, c, d, e, f) \mapsto\left(\frac{q^{-2 n-1} d e f}{b^{2}}, \frac{q^{-n-1} c d e f}{b^{2}}, \frac{q^{-n} f}{b}, \frac{q^{-n} e}{b}, \frac{q^{-n} d}{b}\right), \\
(b, c, d, e, f) \mapsto\left(\frac{q^{-2 n-1} c d e}{b^{2}}, \frac{q^{-n-1} c d e f}{b^{2}}, \frac{q^{-n} c}{b}, \frac{q^{-n} d}{b}, \frac{q^{-n} e}{b}\right), \\
(b, c, d, e, f) \mapsto\left(\frac{q^{-n-1} d e f}{b}, \frac{q^{-n-1} c d e f}{b^{2}}, f, e, d\right),
\end{gathered}
$$

respectively".

6. Replace "Funding: This research received no external funding." with "Funding: R.S.C.-S. acknowledges financial support through the research project PGC2018-09504-B-C33 supported by Agencia Estatal de Investigación of Spain.".

7. In the line before "Conflicts of Interest: ... ", insert "Acknowledgments: H.S.C. would like to thank Mourad Ismail for valuable discussions.".

The authors would like to apologize for any inconvenience caused to the readers by these changes. The changes do not affect the scientific results. The manuscript will be updated, and the original will remain online on the article webpage, with a reference to this correction.

\section{Reference}

1. Cohl, H.S.; Costas-Santos, R.S.; Ge, L. Terminating Basic Hypergeometric Representations and Transformations for the Askey-Wilson Polynomials. Symmetry 2020, 12, 1290. [CrossRef]

Publisher's Note: MDPI stays neutral with regard to jurisdictional claims in published maps and institutional affiliations.

(C) 2020 by the authors. Licensee MDPI, Basel, Switzerland. This article is an open access article distributed under the terms and conditions of the Creative Commons Attribution (CC BY) license (http://creativecommons.org/licenses/by/4.0/). 